\title{
Automatic Multi-modal ToF/CT Organ Surface Registration
}

\author{
Kerstin Müller $^{1}$, Sebastian Bauer ${ }^{1}$, Jakob Wasza ${ }^{1}$, Joachim Hornegger ${ }^{1,2}$ \\ ${ }^{1}$ Pattern Recognition Lab, Friedrich-Alexander University Erlangen-Nuremberg \\ ${ }^{2}$ Erlangen Graduate School in Advanced Optical Technologies (SAOT) \\ kerstin.mueller@informatik.uni-erlangen.de
}

\begin{abstract}
In the field of image-guided liver surgery (IGLS), the initial registration of the intra-operative organ surface with preoperative tomographic image data is performed on manually selected anatomical landmarks. In this paper, we introduce a fully automatic scheme that is able to estimate the transformation for initial organ registration in a multi-modal setup aligning intra-operative time-of-flight ( ToF) with preoperative computed tomography (CT) data, without manual interaction. The method consists of three stages: First, we extract geometric features that encode the local surface topology in a discriminative manner based on a novel gradient operator. Second, based on these features, point correspondences are established and deployed for estimating a coarse initial transformation. Third, we apply a conventional iterative closest point (ICP) algorithm to refine the alignment. The proposed method was evaluated for an open abdominal hepatic surgery scenario with invitro experiments on four porcine livers. The method achieved a mean distance of $4.82 \pm 0.79 \mathrm{~mm}$ and $1.70 \pm 0.36 \mathrm{~mm}$ for the coarse and fine registration, respectively.
\end{abstract}

\section{Introduction}

Image-guided surgery techniques augment the anatomical expertise of the surgeon with a patient-specific source of information by correlating the operative field with preoperative tomographic image data. This allows the physician to see the surgical probe position in relation to anatomical structures during the procedure. Benefits of the integration of computer navigated tool guidance for hepatic surgery are (i) the enhancement of the resection of subsurface targets and avoidance of critical structures, (ii) improved outcomes due to reduced resection margins, and (iii) an expansion of the spectrum of resectability [1]. The essential step in image-guided surgery is the determination of a mapping between the intra-operative presentation of the exposed organ (physical space) and the patient anatomy available from pre-operatively acquired tomographic data (image space). The current registration protocol for image guidance in hepatic surgery is based on a landmark-based initial alignment. As a prerequisite, anatomical fiducials are manually selected in the preoperative image sets prior to surgery [1]. 
Then, during the procedure, the homologous physical-space locations are digitized with a pen probe system. Last, the initial landmark-based registration is refined by conventional rigid surface registration techniques [2]. In clinical practice, manual fiducial selection by radiology experts is difficult, subjective and time-consuming. In this paper, we introduce a fully automatic scheme that is able to estimate the transformation for initial organ registration and ICP initialization without any manual interaction. The evaluation considers a multi-modal $\mathrm{ToF} / \mathrm{CT}$ setup for IGLS, where the intra-operative surface data is acquired with a ToF camera. The applicability of ToF technology for intra-operative surface acquisition was investigated by Seitel et al. [3], achieving promising results on a variety of porcine organs.

\section{Materials and Methods}

The proposed registration framework is composed of three stages (Fig. 1). First, ToF and CT data are preprocessed and transformed into a surface mesh representation. Second, we extract local surface feature descriptors from both data sets. Third, in order to register the surfaces, point correspondences are established by feature matching and a rigid body transformation is estimated. This coarse registration can then be refined with conventional ICP variants.

\subsection{ToF/CT Surface Mesh Generation}

ToF imaging directly acquires metric 3D surface information in real-time with a single sensor based on the phase shift between an actively emitted and the reflected optical signal. The measurements of the ToF camera can be represented as a set of points or vertices $\mathcal{V}=\left\{v_{i}\right\}, i \in\{1, \ldots, w \cdot h\}$, where $v_{i} \in \mathbb{R}^{3}$ denotes the vertex coordinates, $w \times h$ the sensor resolution. In consideration of the inherent noise in ToF range measurements and w.r.t. the trade-off between data denoising and preservation of topological structure, we perform data preprocessing in a way that gives priority to the topological reliability of the surface. In particular, we combine temporal averaging with edge-preserving median and bilateral filtering. In terms of segmentation, for the in-vitro experiments (Sect. 2.5), we used a semi-automatic scheme. The organ is extracted by thresholding and

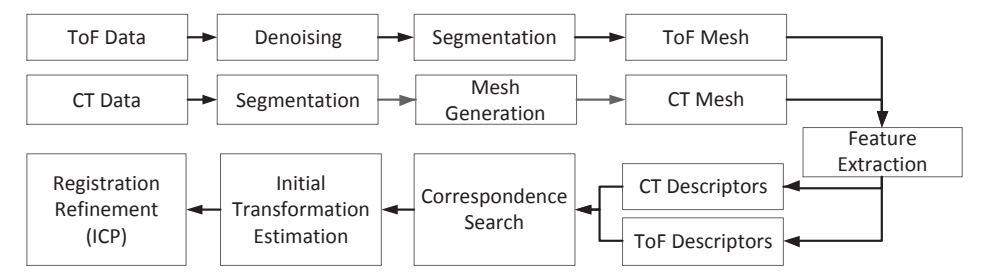

Fig. 1. Data processing flowchart of the proposed framework for feature-based multimodal ToF/CT organ registration. 
the remaining point cloud triangulated, providing the intra-operative ToF surface mesh. The anatomical reference surface is segmented from preoperative CT data using a region growing framework with manual seed point placement. Subsequently, we apply the marching cubes algorithm on the extracted binary volumetric segmentation mask and eventually decimate the dense CT mesh.

\subsection{Local Surface Descriptors}

In this section, we introduce a gradient operator that computes a numerical differentiation of a geometric scalar field defined on a $2 \mathrm{D}$ manifold. Then, we present descriptors that encode the spatial distribution and orientation of the resulting gradient vector field within the local neighborhood of a mesh vertex.

Geometric CUSS Gradient Conventionally, in a 2D image, gradients are computed by differentiating scalar data in two orthogonal directions. For 2D manifolds, we propose a novel gradient operator $\nabla f\left(v_{i}\right)$ that is based on a circular uniform surface sampling (CUSS) technique being invariant to mesh representation and density [4]. Given is a scalar field $f\left(v_{i}\right)$ that holds a 1D geometric feature for every mesh vertex $v_{i} \in \mathcal{V}$, e.g. the signed distance of a point to the best fitting plane of its neighborhood. In a first step, the tangent plane $T_{i}$ being defined by the corresponding normal $n_{i}$ is determined for the vertex $v_{i}$. Next, a circular uniform sampling of $T_{i}$ is performed via rotating a reference vector $a_{i} \in T_{i},\left\|a_{i}\right\|_{2}=1$ around $n_{i}$ by the angles $\phi_{s}=s \cdot \frac{2 \pi}{N_{s}}, s \in\left\{1, \ldots, N_{s}\right\}$, yielding $R_{\phi_{s}} a_{i}$. The circular sampling density is given by $N_{s}, R_{\phi_{s}}$ denotes the $3 \times 3$ rotation matrix for $\phi_{s}$. Scaling the vectors $R_{\phi_{s}} a_{i}$ with a sampling radius $r_{s}$ provides a set $\mathcal{P}$ of points $p_{s} \in T_{i},|\mathcal{P}|=N_{s}$

$$
\mathcal{P}=\left\{p_{s} \mid p_{s}=v_{i}+r_{s} \cdot R_{\phi_{s}} a_{i}\right\}
$$

Finally, the surface sampling is performed by intersecting the mesh with rays that emerge from the points $p_{s}$ and are directed parallel to $n_{i}$ (Fig. 2(a)). The intersection points are denoted $m_{s}$, the scalar field value $f\left(m_{s}\right)$ is interpolated w.r.t. the adjacent vertices. The CUSS gradient $\nabla f\left(v_{i}\right)$ at the vertex $v_{i}$ can then be expressed as

$$
\nabla f\left(v_{i}\right)=\frac{1}{N_{s}} \sum_{s=1}^{N_{s}} \frac{f\left(m_{s}\right)-f\left(v_{i}\right)}{\left\|m_{s}-v_{i}\right\|_{2}} \cdot R_{\phi_{s}} a_{i}
$$

\subsection{Descriptor Encoding}

For each vertex $v_{i}$, the descriptor extracts the spatial distribution and orientation of the CUSS gradient vector field (Fig. 2(b)) for the local set of vertices $\hat{\mathcal{V}} \subset \mathcal{V}$ that reside within a spherical volume of interest. First, the gradient vectors $\nabla f\left(v_{i}\right)$ are projected onto the three planes of a local coordinate system. It is spanned by the vertex normal $n_{i}$ and a second axis $m_{i} \in T_{i}$ pointing 
into the dominant gradient direction. Second, for each plane, the projected vectors are separated into circular segments and binned in polar histograms [5]. The concatenation of these histograms yields the descriptor that is invariant to translation and rotation but not invariant to scale, as we incorporate the metric scale of the surface topology as an important characteristic.

\subsection{Correspondence-based Registration}

For the registration of ToF and CT data $\left(\mathcal{V}_{1}, \mathcal{V}_{2}\right)$, the corresponding sets of local descriptors $\mathcal{D}_{1}, \mathcal{D}_{2}$ are computed first. Based on these descriptor sets, point correspondences are established between $\mathcal{V}_{1}$ and $\mathcal{V}_{2}$ by searching the mutual best match with an Euclidean similarity metric. In order to eliminate false correspondences, the set of point pairs is decimated by comparing all internal pairwise distances of the two correspondence point sets [6]. The remaining set of correspondences (Fig. 2(c)) is used to estimate a rigid body transformation. Last, based on this initial transformation, the registration is refined with an ICP variant [2].

\subsection{Experiments}

The proposed method was evaluated with in-vitro experiments on four porcine livers. ToF data were acquired with a CamCube 2.0 (PMDTechnologies GmbH) at a distance of $60 \mathrm{~cm}$. CT data were acquired with an Artis zeego C-arm system (Siemens AG, Healthcare Sector). The livers were scanned with a resolution of $512 \times 512 \times 348$ voxels and a spacing of $0.70 \times 0.70 \times 0.70 \mathrm{~mm}$.

\section{Results}

Quantitative ToF/CT registration results of the four porcine livers are given in Fig. 3. In order to evaluate the registration accuracy, we measured the residual mean distance $d$ of all ToF surface points to the closest CT surface points.

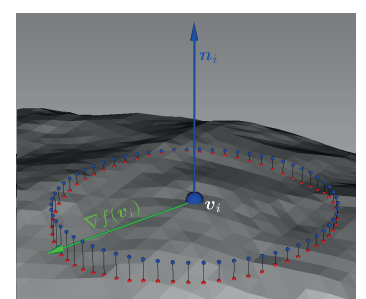

(a)

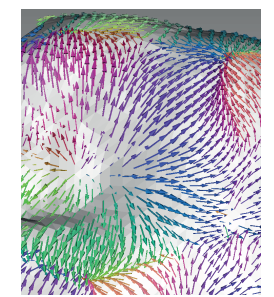

(b)

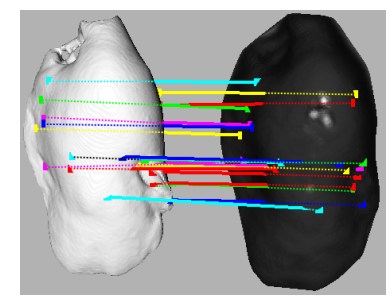

(c)

Fig. 2. (a) Circular uniform surface sampling scheme. (b) CUSS gradient vector field, the orientation is additionally color-coded w.r.t. the global coordinate system. (c) Point correspondences established by feature matching, a subset (15\%) is shown. 
Fig. 3. Left: Qualitative alignment after initial registration (left) and ICP refinement (middle), all distances in mm (right); Right: Quantitative results for livers L1-L4.
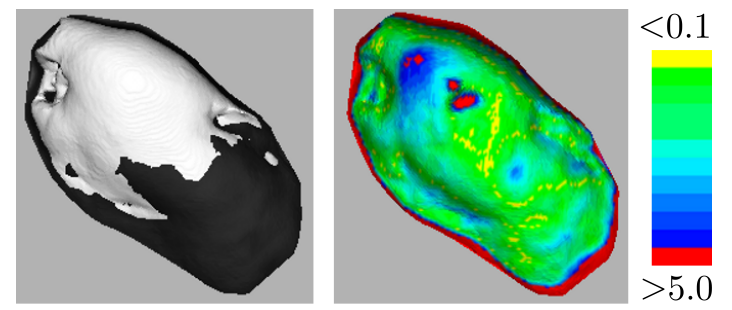

\begin{tabular}{lll}
\hline & $d_{\text {init }}[\mathrm{mm}]$ & $d_{\text {fine }}[\mathrm{mm}]$ \\
\hline L1 & 5.99 & 1.57 \\
L2 & 4.35 & 2.22 \\
L3 & 4.60 & 1.65 \\
L4 & 4.34 & 1.37 \\
\hline Mean & $4.82 \pm 0.79$ & $1.70 \pm 0.36$ \\
\hline
\end{tabular}

The proposed method achieved a mean distance between the ToF/CT mesh of $d_{\text {init }}=4.82 \pm 0.79 \mathrm{~mm}$ for the initial coarse registration and $d_{\text {fine }}=1.70 \pm$ $0.36 \mathrm{~mm}$ for the subsequent ICP refinement. The descriptor parameters were set heuristically. Qualitative registration results for liver L2 are shown in Fig. 3. The runtime of the intra-operative pipeline (preprocessing, feature extraction, correspondence search, registration) is less than $30 \mathrm{~s}$ on a $2.66 \mathrm{GHz} \mathrm{CPU}$, with the correspondence search running on a NVIDIA Geforce 8400 GPU. CT data preprocessing can be performed pre-operatively.

\section{Discussion}

We have presented a multi-modal rigid registration framework for estimating the initial alignment of an exposed intra-operative organ surface with preoperative data based on local surface descriptors. Preliminary experimental results on porcine liver data are encouraging and suggest that a feature-based correspondence search can replace the manual selection of anatomical landmarks for ICP initialization in organ registration applications (e.g. IGLS). Ongoing work analyzes the influence of the ToF preprocessing pipeline and the robustness of the descriptor w.r.t. minor deformations.

\section{References}

1. Cash D, Miga M, Glasgow S, et al. Concepts and preliminary data toward the realization of image-guided liver surgery. J Gastrointest Surg. 2007;11(7):844-59.

2. Besl J, Neil M. A method for registration of 3D shapes. IEEE Trans Pattern Anal Mach Intell. 1992;14(2):239-56.

3. Seitel A, dos Santos TR, Mersmann S, et al. Time-of-Flight Kameras für die intraoperative Oberflächenerfassung. In: Proc BVM; 2010. p. 11-5.

4. Bauer S, Wasza J, Müller K, et al. 4D photogeometric face recognition with timeof-flight sensors. In: Proc WACV; 2011, accepted for publication.

5. Zaharescu A, Boyer E, Viranasi K, et al. Surface feature detection and description with applications to mesh matching. In: Proc CVPR; 2009. p. 373-80.

6. Gelfand N, Mitra N, Guibas L, et al. Robust global registration. In: Proc SGP; 2005. p. 197-206. 